\title{
Soft computing approaches for next-generation sustainable systems (SCNGS)
}

\author{
Pasumpon Pandian ${ }^{1} \cdot$ Xavier Fernando ${ }^{2} \cdot$ Tomonobu Senjyu $^{3}$
}

Published online: 25 February 2019

(C) Springer-Verlag GmbH Germany, part of Springer Nature 2019

Soft computing provides the better solution for some computational issues like NP-hard problem and their applications applied to sustainable computing systems, which helps to resolve the problems of energy consumption, fuel reduction, delay tolerance, loss rate and global warming. The most integration models of soft computing techniques are fuzzy logic, deep learning, evolutionary computation, optimization algorithm, metaheuristics, Bayesian networks, expert systems, perceptron, differential algorithms, pattern recognition and reasoning models. Sustainable systems addressed the solution for power consumption in soft computing approaches and help to provide efficient solutions for the problem-solving methods. Soft computing gives beneficial use in the future applications of sustainable systems.

Notably, researcher uses the soft computing approaches in data mining, machine learning, sustainable computing, capsule networks, neural networks and fuzzy linear systems to provide the intelligent solutions for the real-life problems and that are related to the sustainable systems. The goal of this special issue is to bring the theoretical preparations and practical applications of research contribution in all aspects of soft computing applications applied to sustainable computing. Further, it validates through the soft computing models in terms of precision, uncertainty, partial truth and approximation analysis.

This special issue features some of the articles related to pattern recognition applications and includes saliency detection, gender classification, biotic cross-pollination and multimodal biometric authentication for optimized results. Next, some of the article discussed the optimization algorithms in neural networks, vehicular networks and multimedia applications. Soft computing models enhanced the evolution of the Internet of things (IoT) in smart systems for secure and efficient communication. Remaining articles explained that the process of fuzzy-neuro model in soft computing and it states that the process of fuzzy entropy and hybrid data fusion techniques.

\section{Compliance with ethical standards}

Conflict of interest The authors declare that they have no conflict of interest.

Publisher's Note Springer Nature remains neutral with regard to jurisdictional claims in published maps and institutional affiliations.

Pasumpon Pandian

pasumponpandian32@gmail.com

Xavier Fernando

fernando@ee.ryerson.ca

Tomonobu Senjyu

senjyu@cc.u-ryukyu.ac.jp

1 Department of CSE, Vaigai College of Engineering, Madurai, India

2 Department of Electrical and Computer Engineering, Ryerson University, Toronto, Canada

3 Department of Engineering, University of the Ryukyus, Nishihara, Japan 